\title{
BMJ Open Quality Project Console: a quality improvement initiative for neonatal abstinence syndrome in a children's hospital level IV neonatal intensive care unit
}

\author{
Kathryn L Ponder ำ , ${ }^{1}$ Charles Egesdal, ${ }^{2}$ Joanne Kuller, ${ }^{1}$ Priscilla Joe ${ }^{1}$
}

To cite: Ponder KL, Egesdal C, Kuller J, et al. Project Console: a quality improvement initiative for neonatal abstinence syndrome in a children's hospital level IV neonatal intensive care unit. BMJ Open Quality 2021;10:e001079. doi:10.1136/ bmjoq-2020-001079

- Additional supplemental material is published online only. To view, please visit the journal online (http://dx.doi.org/10. 1136/bmjoq-2020-001079).

Received 22 June 2020 Revised 10 April 2021 Accepted 19 April 2021

\section{Check for updates}

(c) Author(s) (or their employer(s)) 2021. Re-use permitted under CC BY-NC. No commercial re-use. See rights and permissions. Published by BMJ.

${ }^{1}$ Neonatology, UCSF Benioff Children's Hospital Oakland, Oakland, California, USA

${ }^{2}$ Pediatrics, UCSF Benioff Children's Hospital Oakland, Oakland, California, USA

Correspondence to Dr Kathryn L Ponder; Kathryn.Ponder@ucsf.edu

\section{ABSTRACT}

Objective To improve care for infants with neonatal abstinence syndrome.

Design Infants with a gestational age of $\geq 35$ weeks with prenatal opioid exposure were eligible for our quality improvement initiative. Interventions in our PlanDo-Study-Act cycles included physician consensus, re-emphasis on non-pharmacological treatment, the Eat Sleep Console method to measure functional impairment, morphine as needed, clonidine and alternative soothing methods for parental unavailability (volunteer cuddlers and automated sleeper beds). Pre-intervention and postintervention outcomes were compared.

Results Length of stay decreased from 31.8 to 10.5 days $(p<0.0001)$ without an increase in readmissions. Composite pharmacotherapy exposure days decreased from 28.7 to $5.5(p<0.0001)$. This included reductions in both morphine exposure days $(p<0.0001)$ and clonidine exposure days $(p=0.01)$. Fewer infants required pharmacotherapy $(p=0.02)$.

Conclusions Our study demonstrates how a comprehensive initiative can improve care for infants with neonatal abstinence syndrome in an open-bay or a highacuity neonatal intensive care unit when rooming-in is not available or other comorbidities are present.

\section{INTRODUCTION}

Neonatal abstinence syndrome (NAS) is a withdrawal syndrome caused by the abrupt cessation of in utero drug exposure once a neonate is born and typically refers to opioid withdrawal. The incidence of NAS has grown nearly fivefold in the last decade and is associated with other comorbidities, including low birth weight and feeding difficulties. ${ }^{1}$ In the USA, the mean length of stay (LOS) is 16.9 days for infants with NAS and 23 days for those requiring pharmacotherapy, contributing to substantial healthcare costs, particularly for Medicaid. ${ }^{1}$ It also interferes with parent-infant bonding and attachment. ${ }^{1}$ Pharmacotherapy to treat NAS contributes to prolonged hospital stays and has largely unknown long-term developmental consequences, with concerns for possible adverse outcomes. $^{23}$

\section{Problem}

Our institution's LOS for NAS was substantially above the national average, ${ }^{1}$ at 31.8 days. We felt this could be related to a number of factors, including some unique challenges associated with being a level IV neonatal intensive care unit (NICU) within a free-standing children's hospital, where all patients are outborn. For instance, parental rooming-in ${ }^{4}$ and increased parental presence $^{6}$ both reduce LOS and need for pharmacotherapy for NAS. However, as an open-bay NICU in a children's hospital without private rooms, parental rooming-in is not available in our institution. Second, the transfer of these infants could occasionally incur substantial travel distances and therefore create geographical barriers for families. Finally, infants are often transferred from other institutions either due to the severity of their NAS symptoms or due to other comorbidities such as respiratory distress or congenital infections, factors which may increase the risk of prolonged hospitalisation.

\section{Available knowledge}

Published quality improvement (QI) reports in the last 5 years suggest that an emphasis on non-pharmacological care bundles with empowering parental messaging, ${ }^{2478}$ measures of infant functional impairment to guide pharmacotherapy, ${ }^{4-10}$ and pharmacotherapy weaning protocols ${ }^{11}$ may shorten LOS and pharmacotherapy use for NAS. These principles guided our Plan-Do-Study-Act (PDSA) cycles, with some adaptations to our unique set of challenges.

\section{Specific aim}

In December 2018, our NICU developed a QI initiative, named Project Console, to address the unique challenges of managing NAS in our unit, with a global goal of improving care for infants with NAS. Our specific aim was that 'by January 2020, we will: (1) reduce the 
average LOS by $50 \%$ and (2) reduce the average number of pharmacotherapy exposure days by $50 \%$, in infants with a gestational age of $\geq 35$ weeks who are admitted to the NICU with prenatal opioid exposure and are at risk of NAS (excluding infants with significant comorbidities including major cardiac/abdominal surgeries or those requiring extracorporeal life support (ECLS), inhaled nitric oxide (iNO) or high-frequency oscillatory ventilation (HFOV)).'

\section{METHODS}

\section{Context and population}

From December 2018 to January 2020, we conducted a QI project in an urban, level IV NICU in the USA. We are a licensed 37-bed open-bay NICU within a free-standing children's hospital. The NICU has approximately 390 admissions per year. All admissions are outborn, either transferred from a referring hospital or admitted via the emergency department (ED). Infants with NAS are admitted to the NICU and not to the paediatric ward. Patients with NAS make up a small proportion of total NICU admissions, approximately 5-11 infants per year. Eighty-five per cent of patients have public health insurance and $15 \%$ have private insurance. Our NICU serves a population that is approximately $36 \%$ Hispanic or Latino, 29\% White or Caucasian, $18 \%$ Black or African American, $14 \%$ Asian, $<1 \%$ Hawaiian or Pacific Islander, and $<1 \%$ Native American infants. We are a teaching hospital, with multidisciplinary teams contributing to the care for infants with NAS.

\section{Pre-intervention}

During the baseline period of January 2015-November 2018, our NICU followed a written NAS guideline developed by the neonatology group. Pharmacotherapy initiation and escalation were based on a total Finnegan tool score, whereby three scores $\geq 8$, two scores $\geq 12$ or one score $\geq 15$ prompted the initiation of scheduled morphine every 3 hours. Clonidine was used as an adjunctive agent, and phenobarbital as a third agent if needed. Non-pharmacological care suggestions were included in a written guideline available to the neonatologists.

\section{Interventions}

We identified four key drivers of LOS and pharmacotherapy exposure: a guideline for the use of nonpharmacological and pharmacological treatment strategies, staff education on non-pharmacological management, parental empowerment and involvement, and alternative soothing methods for parental unavailability (figure 1). Interventions were developed and implemented over 3 PDSA cycles.

\section{PDSA CYCLE 1: DECEMBER 2018 \\ Physician discussion and consensus}

A group of 25 neonatologists provide coverage for our unit. A literature review and presentation on NAS was conducted as part of a regularly scheduled educational meeting. Initial guideline revisions were discussed, including alternative pharmacotherapy options, criteria for selective toxicology testing of infants, discharge
SMART AIMS

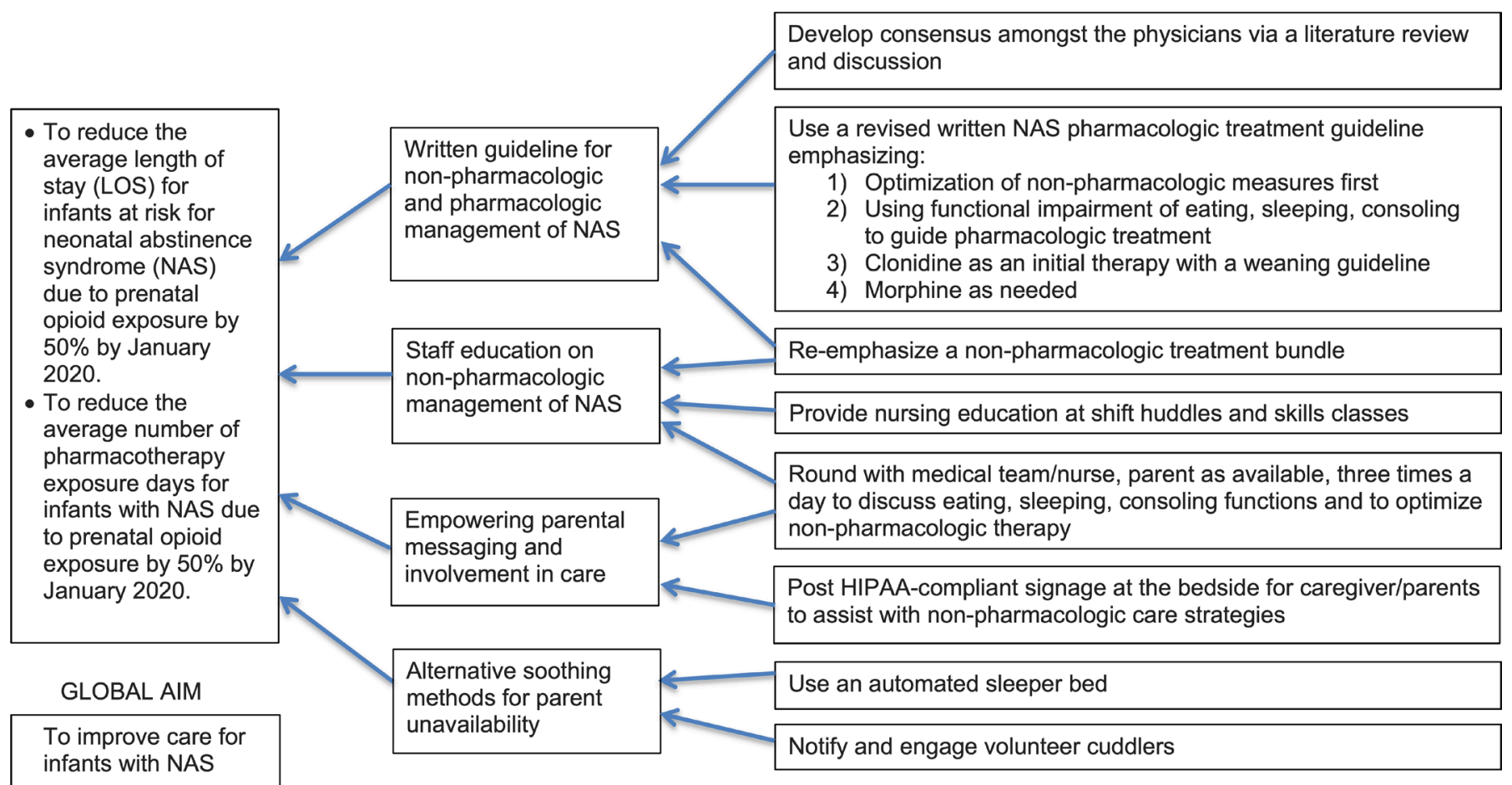

Figure 1 Key driver diagram for Project Console. HIPAA, Health Insurance Portability and Accountability Act of 1996. 
criteria including observation of the infant for 48 hours after the last medication dose, and recommendations for close primary care physician follow-up within 1 week of discharge. Discussions continued via electronic correspondence.

\section{Eat Sleep Console}

Traditional Finnegan scoring has known limitations including scores for yawning and sneezing contributing to an overall score that could prompt pharmacotherapy use. ${ }^{12}$ The total score and variability of a Finnegan score will increase over time even in non-exposed infants with normal developmental changes. ${ }^{13}$ Instead of relying on a Finnegan score, we transitioned to the Eat Sleep Console model, ${ }^{89}$ an assessment method which prioritises measures of an infant's functional impairment to guide treatment. Finnegan scores were still performed a few times a day to determine nursing staffing and acuity level but were not used to make treatment decisions. The medical team continued rounding at the bedside three times a day (morning, evening and overnight) that involved the bedside nurse or charge nurse, and a parent if available, in addition to more informal communication throughout the day for any concerns. Nurses charted their assessments in their plan of care in the electronic medical record. Our guideline was rewritten to consider the initiation of pharmacotherapy after a team huddle if nonpharmacological care had been optimised first, non-NAS causes were excluded (ie, poor latch due to anatomical factors, prematurity with feeding, other comorbid conditions) and the baby continued to show functional impairment defined as: (1) poor feeding due to NAS (unable to sustain feeding for $10 \mathrm{~min}$ or take an age-appropriate volume), OR (2) sleeping $<1$ hour after feeding due to NAS, OR (3) the inability to be consoled in $10 \mathrm{~min}$.

\section{First pharmacotherapy guideline revision: morphine as needed}

When non-pharmacological measures were being optimised but an infant could not easily feed, sleep or be consoled, then morphine was recommended as an initial pharmacotherapy strategy, using a dose of $0.04 \mathrm{mg} /$ $\mathrm{kg} /$ dose by mouth every 3 hours as needed. The guideline recommended escalating to scheduled doses of morphine if eating/sleeping/consoling functions were not improving. Clonidine was the recommended secondline agent.

\section{Emphasis on volunteers}

Our NICU already had a well-established unit-specific volunteer cuddler programme in place. For this initiative, we had an increased communication effort with the volunteer department to notify them when a patient with NAS was admitted and to prioritise infants with NAS for this programme. Our NICU leadership provided frequent feedback to the volunteers to maintain their engagement with the project.

\section{PDSA CYCLE 2: FEBRUARY 2019}

Re-emphasis on a non-pharmacological care bundle with parental messaging and staff education

Nursing staff received education on NAS at change of shift huddles and nursing skills classes, with an emphasis on using our non-pharmacological care bundle and encouraging parental involvement. The bundle was developed into a corresponding one-page, Health Insurance Portability and Accountability Act of 1996-compliant sign that could be posted at the bedside for parents and staff to reference or used when counselling parents (see online supplemental material 1), as well as a bedside tip sheet for nurses. Nursing feedback was discussed by NICU leadership regularly. Components of the non-pharmacological care bundle included:

1. Caregiver contact: encourage parents (or foster parents/volunteers) to visit and hold the baby. This could include skin-to-skin when held by parents, swaddling with the infant's hands near his or her mouth, and/or use of non-nutritive sucking with a pacifier.

2. Environment: maintain a quiet, low-light environment. Limit the number of visitors. Only one stimulus at a time (ie, do not walk or sway while feeding). Use a swing, but stop if the infant is appearing overstimulated.

3. Feeding: on-demand. Encourage breastfeeding/lactation consultation if eligible (maternal methadone/ buprenorphine exposure is okay if no other contraindications). If using formula, consider lactosereduced or a partially hydrolysed protein formula. Consider fortifying to $22 \mathrm{kcal} /$ ounce after day of life (DOL) 2-3 if there is greater than expected weight loss.

4. Skin: use diaper cream on day 1 to prevent/treat diaper dermatitis. Treat all skin excoriation due to tremors.

\section{Second pharmacotherapy guideline revision:} clonidine \pm morphine as needed

We soon realised that the majority of infants admitted with NAS were receiving pharmacotherapy prior to transfer. At admission, non-pharmacological measures were tried first. If a patient failed non-pharmacological measures, then pharmacotherapy was started. With the next PDSA cycle, we adjusted our pharmacotherapy guideline to use scheduled clonidine as an initial pharmacological treatment strategy, still with the option for as needed doses of morphine. There is increasing evidence for the safety and efficacy of clonidine for infants with NAS. ${ }^{214-19}$ We recommended doses of: $1 \mu \mathrm{g} / \mathrm{kg} /$ dose by mouth every 4 hours. If the infant continued to demonstrate functional impairment, then the guideline recommended that the physician add morphine: $0.04 \mathrm{mg} / \mathrm{kg} /$ dose by mouth every 3 hours as needed. Once functional ability to eat, sleep and be consoled was improved for 24 hours, we recommended attempting to wean the clonidine dose to $0.5 \mu \mathrm{g} / \mathrm{kg} /$ dose by mouth every 4 hours, then lengthening the interval time to every 8 hours as tolerated, then discontinue. 
PDSA CYCLE 3: JUNE 2019

\section{Automated sleeper beds}

A commercially available automated sleeper bed was added to the non-pharmacological care bundle. This was used to assist with sleep and soothing when parents, nursing, volunteer cuddlers or foster parents were unavailable. The sleeper bed automatically responds to infant crying with increasing levels of white noise or swaying movements to soothe the infant, with a time-out feature that discontinues the interventions for persistent crying. We set the sleeper bed to discontinue below the maximal available setting as a conservative measure to ensure safety. We revised our guideline for physicians or neonatal nurse practitioners to order the bed on admission.

\section{Study of the interventions}

The baseline data from our institution were compiled as part of our contribution to the Children's Hospital Network Database, a database of 220 children's hospitals in the USA. We extracted data for infants admitted to our institution between January 2015 and November 2018 and compared these with data we collected during the post-intervention time period for infants admitted between December 2018 and January 2020. An infant was determined to have a clinical diagnosis of NAS as determined by a physician, based on prenatal opioid exposure history (either to illicit drugs or medication as part of a maternal treatment programme) and classic symptomatology. Infants were excluded from analyses in our study for a gestational age $<35$ weeks or what we felt to be a significant comorbidity that would make it difficult to ascertain whether differences in LOS or sedation medication use were due to NAS-related reasons, including need for ECLS, major invasive cardiac/abdominal surgery, iNO or HFOV use. We compared pre-intervention and postintervention maternal and infant demographic information to evaluate whether these factors could have contributed to differences in our outcomes. We also compared pre-intervention and post-intervention process measures, balancing measures and outcomes.

\section{Measures}

Our primary outcome was LOS, defined by the number of days between the date of admission to our NICU to the date of discharge. Our secondary outcomes were composite pharmacotherapy exposure days, morphine exposure days and clonidine exposure days, defined as the number of calendar days an infant received one or more doses of morphine and/or clonidine during the hospitalisation in our NICU. Process measures included whether the infant was discharged with a biological parent versus foster care and whether the infant was receiving any breast milk at the time of discharge. Balancing measures were the number of infants discharged home on pharmacotherapy, seizures, readmissions for any reason within 30 days, as well as readmissions for NAS within 30 days from the day of discharge.

\section{Analysis}

We used $\mathrm{X}^{2}$ tests for categorical variables and two-tailed $\mathrm{t}$-tests for continuous variables, using $\mathrm{p}<0.05$ to indicate statistical significance. We used statistical process control (SPC) charts to evaluate the impact of our interventions over time and to determine when special cause variation occurred, as indicated by eight consecutive data points above or below the centre line. ${ }^{20}$ All analyses were conducted using Microsoft Excel QIMacros.

\section{RESULTS}

Twenty-four infants met criteria for inclusion in the baseline pre-intervention group. Twelve infants met inclusion criteria for the post-intervention group. Demographics comparing pre-intervention and post-intervention groups are shown in table 1 . There were no differences in gestational age, birth weight, per cent on public insurance, per cent admitted from home via the ED, DOL at admission, or race/ethnicity between the study periods. There were significantly more male infants in the preintervention group compared with the post-intervention group ( $p=0.04)$. The majority of infants were admitted to our unit having already received pharmacotherapy for NAS at the referral hospital, which did not differ between the groups. Fifty per cent of infants across the pre-intervention and post-intervention time periods were referred due to at least one comorbidity aside from NAS, which did not differ between the groups.

Methadone use was common in the maternal population, but did not rule out concomitant use of other substances including combinations of illicit substances, prescription opioid medications or marijuana products, based on available history or toxicology testing that had been performed prior to admission. Given that maternal and infant toxicology testing has several limitations, we felt the most clinically useful variable regarding maternal drug use was to examine whether a mother was receiving MAT (medication-assisted treatment) as part of a treatment programme versus not. In the pre-intervention group, $75 \%$ of mothers were receiving MAT compared with just $41.7 \%$ in the post-intervention group, which was trending towards significance (table $1, \mathrm{p}=0.05$ ). Two of the 18 mothers in MAT in the pre-intervention group (11.1\%) and 2 of the 5 mothers in MAT in the post-intervention group (40\%) received buprenorphine, while the remainder received methadone.

Discharge disposition information is included in table 1. The majority of infants in our study were discharged to foster care. The post-intervention group had a larger percentage discharged to foster care $(75 \%)$ than the preintervention group $(54.2 \%)$, but this was not statistically significant. Volunteer holding hours were not tracked in the pre-intervention group, but averaged 35.4 hours per infant admission in the post-intervention group (ranging from 7 to 82 hours/infant, $n=9$ with available data). Though not designed to test for statistical significance, we noted that Black/African American infants were 
Table 1 Demographics and outcomes pre-intervention and post- intervention

\begin{tabular}{|c|c|c|c|}
\hline Demographic/outcome & $\begin{array}{l}\text { Pre-intervention, } \mathbf{n = 2 4} \\
\text { n (\%) or mean (range) }\end{array}$ & $\begin{array}{l}\text { Post-intervention, } \mathrm{n}=12 \\
\mathrm{n}(\%) \text { or mean (range) }\end{array}$ & $P$ value \\
\hline Gestational age (weeks) & $38.7(35-42.3)$ & $38.5(36-42.3)$ & 0.78 \\
\hline Birth weight (grams) & 3081.5 (2300.0-4027.0) & $3102.2(2540.0-3785.0)$ & 0.87 \\
\hline Male sex & $20(83.3)$ & $6(50.0)$ & $0.04^{*}$ \\
\hline Public insurance & $24(100.0)$ & $12(100.0)$ & $\mathrm{n} / \mathrm{a}$ \\
\hline Admit from home/ED & $1(4.2)$ & $2(16.7)$ & 0.20 \\
\hline DOL at admission (days) & $6.8(0-33)$ & $8.6(1-30)$ & 0.56 \\
\hline Admitted on pharmacotherapy & $15(62.5)$ & $8(66.7)$ & 0.81 \\
\hline Non-NAS referral reason† & $11(45.8)$ & $7(58.3)$ & 0.48 \\
\hline Infant race/ethnicity $\ddagger$ & & & 0.76 \\
\hline Black or African American & $11(45.8)$ & $5(41.7)$ & \\
\hline White or Caucasian & $10(41.7)$ & $6(50.0)$ & \\
\hline Asian & $0(0)$ & $0(0)$ & \\
\hline Other & $5(20.8)$ & $1(8.3)$ & \\
\hline Hispanic or Latino ethnicity§ & $4(16.7)$ & $1(8.3)$ & \\
\hline Maternal MAT at infant admission $\ddagger$ & $18(75.0)$ & $5(41.7)$ & 0.05 \\
\hline Black or African American & $9 / 11(81.8)$ & $1 / 5(20.0)$ & \\
\hline White or Caucasian & $7 / 10(70.0)$ & $3 / 6(50.0)$ & \\
\hline Other & $4 / 5(80)$ & $1 / 1(100.0)$ & \\
\hline Hispanic or Latino ethnicity§ & $4 / 4(100)$ & $1 / 1(100.0)$ & \\
\hline Home with a biological parentł & $11(45.8)$ & $3(25.0)$ & 0.23 \\
\hline Black or African American & $3 / 11(27.3)$ & $0 / 5(0.0)$ & \\
\hline White or Caucasian & $5 / 10(50.0)$ & $2 / 6(33.0)$ & \\
\hline Other & $3 / 5(60.0)$ & $1 / 1(100.0)$ & \\
\hline Hispanic or Latino ethnicity§ & $2 / 4(50.0)$ & $1 / 1(100.0)$ & \\
\hline Discharged on any breast milk & $6(25.0)$ & $0(0.0)$ & 0.06 \\
\hline Length of stay (days) & $31.8(3-56)$ & $10.5(2-19)$ & $<0.0001^{*}$ \\
\hline Pharmacotherapy exposure (days) & $28.7(0-53)$ & $5.5(0-14)$ & $<0.0001^{*}$ \\
\hline Morphine exposure (days) & $25.0(0-49)$ & $1.4(0-6)$ & $<0.0001^{*}$ \\
\hline Clonidine exposure (days) & $15.1(0-53)$ & $4.6(0-14)$ & $0.01^{*}$ \\
\hline Did not require pharmacotherapy & $2(8.3)$ & $5(41.7)$ & $0.02^{\star}$ \\
\hline Discharged on pharmacotherapy & $2(8.3)$ & $0(0.0)$ & 0.30 \\
\hline Seizure & 0 & 0 & $\mathrm{n} / \mathrm{a}$ \\
\hline Readmitted within 30 days (any reason) & 0 & $1(8.3)$ & 0.15 \\
\hline Readmitted within 30 days due to NAS & 0 & 0 & $\mathrm{n} / \mathrm{a}$ \\
\hline
\end{tabular}

${ }^{*} \mathrm{P}<0.05$ indicates statistical significance.

†Referral reasons included: dysmorphic features $(n=1)$, respiratory distress $(n=3)$, pneumothorax $(n=1)$, congenital infection $(n=4)$, hypoglycaemia $(n=1)$, haematochezia $(n=2)$, pyloric stenosis $(n=1)$, bilious emesis $(n=3)$, ankyloblepharon $(n=1)$, cleft lip/palate $(n=1)$ and supraventricular tachycardia $(n=1)$.

‡May include more than one race/ethnicity.

§Any race.

DOL, day of life; ED, emergency department; MAT, medication-assisted treatment; n/a, not applicable; NAS, neonatal abstinence syndrome.

disproportionately discharged to foster care instead of a biological parent (13 of $16,81.2 \%)$, compared with the percentages of White/Caucasian infants (9 of 16, 56.3\%), other race (2 of 6,33\%), or infants of Hispanic/Latino ethnicity (2 of $5,40 \%$ ) throughout the study period (table 1).
Outcome measures are included in table 1. Our primary outcome of mean LOS significantly decreased from 31.8 to 10.5 days $(p<0.0001)$, a $67 \%$ reduction that exceeded our goal of $50 \%$. Our pharmacotherapy outcome measures also exceeded our goals. Composite pharmacotherapy exposure days significantly decreased from 28.7 


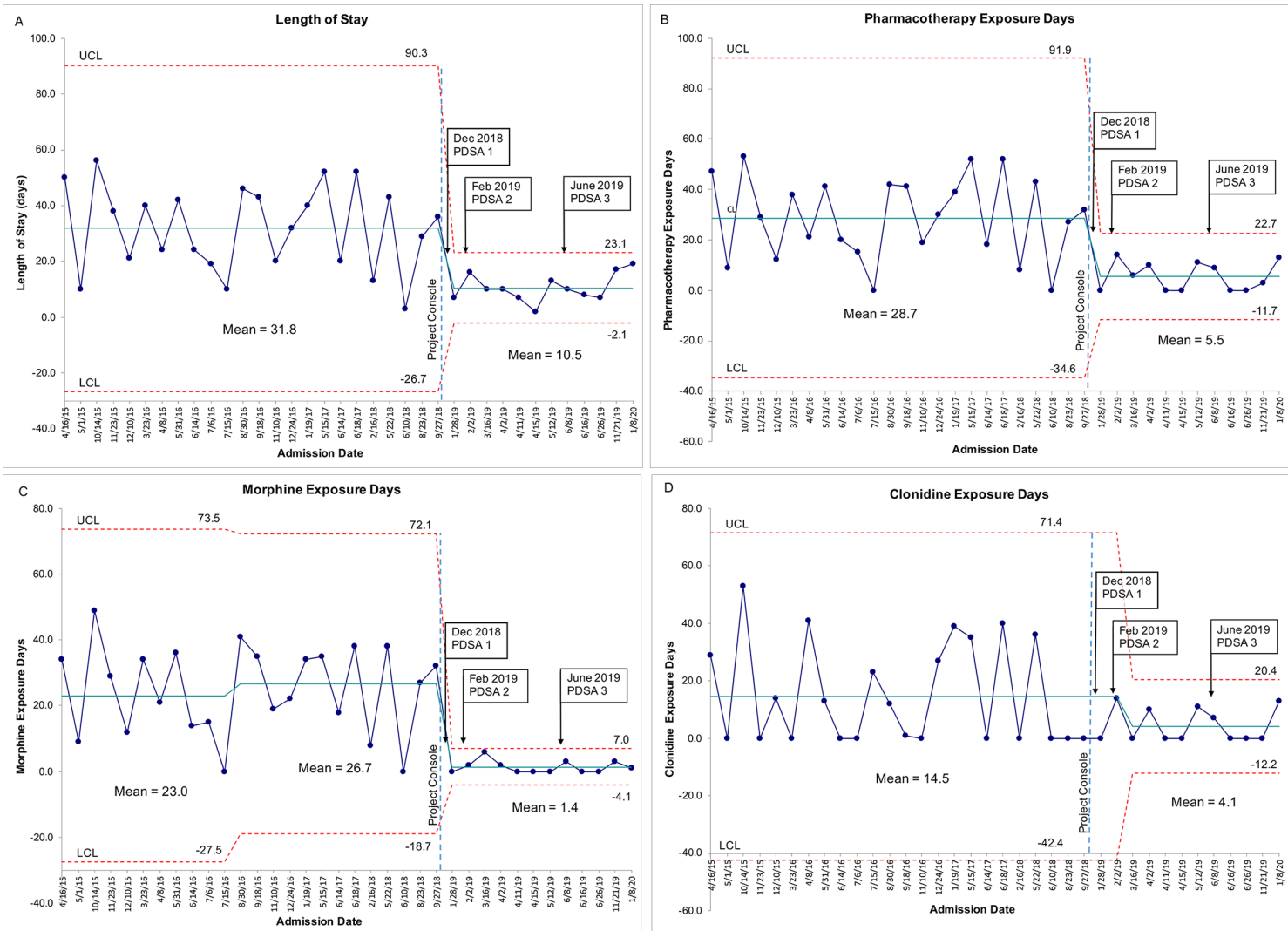

Figure 2 NAS outcomes. Each dot represents an opioid-exposed infant plotted by the date of admission. (A) Length of stay; (B) number of calendar days an infant received pharmacotherapy (at least one dose of morphine and/or clonidine) for NAS during their admission; (C) number of calendar days an infant received morphine during their admission. Special cause variation and a downward shift in the centre lines for A, B and C occurred in January 2019, after the first PDSA cycle was implemented (corresponding to the physician consensus, implementation of Eat Sleep Console, using morphine as needed and an emphasis on volunteers). (D) Number of calendar days an infant received at least one dose of clonidine during their admission. Special cause variation and a downward shift in the centre line for D occurred in March 2019, after the second PDSA cycle was implemented (corresponding to the re-emphasis on a non-pharmacological care bundle with parental messaging and staff education and the second pharmacotherapy guideline revision with use of scheduled clonidine and morphine as needed). LCL lower confidence limit; NAS, neonatal abstinence syndrome; PDSA, Plan-Do-Study-Act; UCL upper confidence limit.

to 5.5 days $(\mathrm{p}<0.0001)$, an $80.8 \%$ reduction. More specifically, there were significant reductions in both morphine exposure days (25 to 1.4 days, $\mathrm{p}<0.0001$, a $94.4 \%$ reduction) and clonidine exposure days (15.1 to 4.6 days, $\mathrm{p}=0.01$, a $69.5 \%$ reduction). The number of infants not requiring any pharmacotherapy after admission to our NICU (ie, treated solely with the non-pharmacological care bundle) improved from $8.3 \%$ pre-intervention to $41.7 \%$ post-intervention $(\mathrm{p}=0.02)$. Two infants in the preintervention group were discharged home on pharmacotherapy with instructions for weaning at home, compared with none in the post-intervention group.

Figure 2 displays the SPC charts for our outcome measures. Special cause variation for LOS (figure 2A), composite pharmacotherapy exposure days (figure 2B) and morphine exposure days (figure 2C) occurred in January 2019, corresponding to the initiation of Project Console and our first PDSA cycle. Special cause variation for clonidine exposure days occurred in March 2019, after our second PDSA cycle was implemented (figure 2D).
The overall degree of variability decreased in all outcome measures after the start of Project Console.

Balancing measures in the pre-intervention and postintervention groups are also listed in table 1 . There were no seizures or readmissions within 30 days due to NAS in either group. There was one readmission in the post-intervention group 24 days after discharge who was ultimately diagnosed with a viral illness. There were no interim symptoms of NAS prior to readmission, nor neurological symptoms at admission to suggest that the infant's symptoms were NAS related.

\section{DISCUSSION}

Our comprehensive QI initiative, Project Console, led to decreased LOS without an increase in adverse events or NAS-related readmissions. We also significantly decreased the days of pharmacotherapy exposure. Despite a transition to using scheduled clonidine when pharmacotherapy was needed, we decreased overall clonidine usage. More infants succeeded with non-pharmacological 
treatment and without the need for any pharmacotherapy in the post-intervention group. Like with other recently published QI initiatives that focus on nonpharmacological care bundles and functionality of the infant's ability to eat, sleep and be consoled, ${ }^{7-10}$ we were surprised by the substantial reduction in both LOS and pharmacotherapy use, reflecting a paradigm shift in the care for infants with NAS at our institution.

There is wide variability in the severity and timing of onset of NAS symptoms in newborns, which we also observed in our study. Lack of breast milk exposure ${ }^{2122}$ has been associated with increased NAS severity. Breast milk exposure was lower in our post-intervention group and thus did not contribute to the improvements we observed. Male infant sex has been associated with increased risk of NAS and NAS requiring pharmacotherapy in one study. ${ }^{23}$ A lower percentage of infants in our post-intervention group were boys. Also, a lower percentage of infants in the post-intervention group were exposed to MAT due to a mother receiving treatment for opioid use disorder. This study was not designed nor powered to analyse subgroup differences in outcome measures. We were reassured, however, that in SPC subgroup analyses (online supplemental material 2A-H), male versus female infants and MAT-exposed versus MAT-unexposed infants exhibited similar decreases in variability and degrees of improvement for LOS and pharmacotherapy exposure days in the post-intervention group. Discharge to foster care has the potential to contribute to an infant's LOS. We found that infants being discharged to foster care had additional hospital days solely due to the wait for placement. A greater percentage of infants were discharged to foster care in our post-intervention group and thus this did not contribute to the improved LOS we observed.

Our approach appears to be safe as indicated by our balancing measures. One small trial indicates no difference in 1-year developmental outcomes for clonidine compared with morphine exposure. ${ }^{19}$ Clonidine's safety and efficacy in infants for the treatment of NAS is promising according to the American Academy of Pediatrics. ${ }^{2}$ Clonidine is an alpha-2 adrenergic agent that has been used to ameliorate drug withdrawal from both opioids and benzodiazepines in adults and older children. ${ }^{14}{ }^{15}$ It acts centrally to block sympathetic nervous system outflow, decreasing the catecholamine release seen with withdrawal and also contributes to a calming effect. ${ }^{215}$ Potential adverse effects include sedation, hypotension and bradycardia, and a risk of a rebound effect with abrupt cessation. For infants, there is increasing favour over phenobarbital for its use as an adjunctive agent in terms of safety. ${ }^{324}$ It has been associated with improved efficacy when used in conjunction with an opioid versus an opioid alone. ${ }^{17}$ One small case series ${ }^{16}$ and one small randomised trial $^{19}$ to date support the safety and efficacy of its use as a primary pharmacotherapy agent in infants with NAS. As with our study, other published QI reports have also had success with using morphine as needed instead of scheduled. $^{810}$
The NAS assessment techniques of both Finnegan Symptom Prioritization ${ }^{47}$ as well as the Eat Sleep Console method $^{7-9}$ that we used have successfully been applied to other recent QI initiatives to gauge NAS severity and guide the need for pharmacotherapy. Many infants in our study could maintain their functional ability to eat, sleep and be consoled after non-pharmacological care was optimised. Finnegan scores were still performed a few times a day in our unit to determine nursing staffing and acuity level but were not used to guide treatment decisions. Acceptability of the Eat Sleep Console scoring method to guide treatment decisions was initially a challenge, but this improved with staff education, an increased comfort level over time, as well as frequent feedback with NICU leadership, ultimately resulting in a large culture shift within the unit.

In addition to a non-pharmacological care bundle, engaging a hospital's volunteer department and using an automated smart sleeper bed when parents or nurses are not available to calm the baby are strategies that could be applied at other institutions. These alternative soothing strategies were effective and thus particularly well received by the staff in our high-acuity unit. Initially, it was challenging to communicate and coordinate with the volunteer department efficiently. Our medical director and clinical nurse specialist led an increased communication effort with this department to notify them when an infant with NAS was admitted. Shift sign-ups were created for the times that parents or caregivers were unavailable, including overnight. Our NICU's experienced, unit-specific volunteers were previously educated in developmental care techniques for NICU patients, likely contributing to the success of their involvement. Inclusion of the automated sleeper bed in the admission orders, as well as a procedure to notify the volunteer department, contributed to the sustainability of these strategies. We feel that frequent feedback to both volunteers and nursing staff increased engagement and likely contributed to the sustainability of the project. For example, Project Console was discussed monthly within our multidisciplinary NICU QI committee. Specifically, project leaders reviewed outcomes, nursing feedback, obstacles and successes, and further opportunities for improvement. We acknowledge a potential limitation of this study is its relatively small size. However, our study uniquely demonstrates how a comprehensive QI initiative can succeed and be sustained in an open-bay or a highacuity NICU when rooming-in is not available or when other infant comorbidities may be present.

Prenatal opioid exposure is inextricably linked with other environmental risks, underscoring the clinical importance of drug exposure as a marker of environmental risk to children. ${ }^{25}{ }^{26}$ We acknowledge that it is not clear if reducing LOS or doses of morphine/clonidine necessarily results in better developmental outcomes and additional studies are needed.

Long-term strategies to improve the care of infants with NAS include improved pre-conception healthcare, and 
the prevention and treatment of opioid use disorder, with a focus on increasing MAT in the community through local and statewide efforts. ${ }^{27}$ The post-intervention group had fewer mothers receiving MAT at the time of the infant's admission and both our pre-intervention and post-intervention groups had high discharge to foster care rates. Downstream effects of more mothers receiving MAT could include decreased discharges to foster care and maintenance of the parent-infant dyad.

Given our high rates of discharge to foster care and our diverse patient population, we felt it was important to further examine this outcome by infant race and ethnicity. We acknowledge this study was not designed to evaluate the statistical significance of this outcome. There is evidence that racial and economic bias, either explicit/ conscious or implicit/unconscious, can affect not only which mothers and infants undergo toxicology testing, but also which families face the consequences of referrals to state agencies, particularly for Black/African American women. ${ }^{289}$ In congruence with the previously mentioned reports, we note that Black/African American infants in our study were disproportionately more likely to be discharged to foster care than to their biological parents compared with White/Caucasian or Hispanic/Latino infants (table 1). Disproportionately fewer mothers of Black/African American infants were receiving MAT in the post-intervention group, and it is unclear if this is due to our small sample size, fewer referrals to MAT, or a lack of successful enrolment and participation after referral. We believe this is an important area of consideration for future QI efforts that focus on treating or preventing NAS. A portion of our revised physician guideline includes standardised criteria for selective toxicology testing for infants with a goal to aid treatment decisions, though we acknowledge that this may not eliminate implicit bias. As a free-standing children's hospital, we found that infant toxicology testing was in almost all cases performed at the referring hospital prior to admission to our institution, also limiting the utility of our attempts to standardise this. The number of infants not requiring any pharmacotherapy and number of infants receiving breast milk were low in our study, thus making it difficult to elaborate on racial differences for these outcomes. Racial equity should be a consideration for future efforts that aim to increase MAT and maintain the parent-infant dyad. ${ }^{27} 3031$

In addition to being better able to maintain the parentinfant dyad, mothers receiving MAT could be eligible to provide breast milk for their infants, which in turn can decrease NAS severity. ${ }^{21} 2232$ Future directions for our programme include working with our obstetrical partners and other resources in the community for NAS prevention, utilisation of MAT for opioid use disorder and improving breast milk availability for those who are eligible.

Acknowledgements The authors would like to acknowledge Dr Matthew Grossman and colleagues for the development of the 'Eat Sleep Console' approach. The authors are grateful to Dr Harvey Karp for his generosity in providing sleeper beds to use in our NICU. The authors would also like to thank Dr Kurlen Payton for his quality improvement expertise and advice. The authors would like to acknowledge Dr Golde Dudell for her role in the development of our local nonpharmacological care guideline, Teresa Proctor, Cindy Collinson, Bette Flushman, developmental staff, volunteers, nurses, social workers, and the families of our patients who play a vital role in the care for these patients.

Contributors KLP prepared the manuscript and literature review and assisted with data collection. CE and PJ collected the data and assisted with revisions of the manuscript. JK led staff education as the neonatal clinical nurse specialist and assisted with revisions of the manuscript. PJ oversaw this initiative and provided support and guidance in the unit as the medical director. All authors read and approved the final paper.

Funding Publication of this project was supported by East Bay Newborn Specialists.

Competing interests None declared.

\section{Patient consent for publication Not required.}

Ethics approval Infants admitted to our NICU with in utero opioid exposure meeting eligibility criteria during the study time period were eligible for the intervention. Our hospital's Institutional Review Board deemed our study as meeting criteria for minimal risk and exempt pursuant to 45 CFR as a quality improvement protocol.

Provenance and peer review Not commissioned; externally peer reviewed.

Data availability statement Data are available upon request.

Supplemental material This content has been supplied by the author(s). It has not been vetted by BMJ Publishing Group Limited (BMJ) and may not have been peer-reviewed. Any opinions or recommendations discussed are solely those of the author(s) and are not endorsed by BMJ. BMJ disclaims all liability and responsibility arising from any reliance placed on the content. Where the content includes any translated material, BMJ does not warrant the accuracy and reliability of the translations (including but not limited to local regulations, clinical guidelines, terminology, drug names and drug dosages), and is not responsible for any error and/or omissions arising from translation and adaptation or otherwise.

Open access This is an open access article distributed in accordance with the Creative Commons Attribution Non Commercial (CC BY-NC 4.0) license, which permits others to distribute, remix, adapt, build upon this work non-commercially, and license their derivative works on different terms, provided the original work is properly cited, appropriate credit is given, any changes made indicated, and the use is non-commercial. See: http://creativecommons.org/licenses/by-nc/4.0/.

ORCID iD

Kathryn L Ponder http://orcid.org/0000-0003-4780-4383

\section{REFERENCES}

1 Patrick SW, Davis MM, Lehmann CU, et al. Increasing incidence and geographic distribution of neonatal abstinence syndrome: United States 2009 to 2012. J Perinatol 2015;35:650-5.

2 Hudak ML, Tan RC, et al, COMMITTEE ON DRUGS. Neonatal drug withdrawal. Pediatrics 2012;129:e540-60.

3 Stefovska VG, Uckermann O, Czuczwar M, et al. Sedative and anticonvulsant drugs suppress postnatal neurogenesis. Ann Neurol 2008;64:434-45.

4 Holmes AV, Atwood EC, Whalen B, et al. Rooming-In to treat neonatal abstinence syndrome: improved family-centered care at lower cost. Pediatrics 2016;137:e20152929.

5 MacMillan KD, Rendon CP, Verma K. Rooming-In for neonatal abstinence syndrome: a systematic review and meta-analysis. JAMA Pediatr 2018;172:345-51.

6 Howard MB, Schiff DM, Penwill N, et al. Impact of parental presence at infants' bedside on neonatal abstinence syndrome. Hosp Pediatr 2017;7:63-9.

7 Wachman EM, Grossman M, Schiff DM, et al. Quality improvement initiative to improve inpatient outcomes for neonatal abstinence syndrome. J Perinatol 2018;38:1114-22.

8 Grossman MR, Berkwitt AK, Osborn RR, et al. An initiative to improve the quality of care of infants with neonatal abstinence syndrome. Pediatrics 2017;139:e20163360.

9 Grossman MR, Lipshaw MJ, Osborn RR, et al. A novel approach to assessing infants with neonatal abstinence syndrome. Hosp Pediatr 2018;8:1-6.

10 Blount T, Painter A, Freeman E, et al. Reduction in Length of Stay and Morphine Use for NAS With the "Eat, Sleep, Console" Method. Hosp Pediatr 2019;9:615-23. 
11 Hall ES, Wexelblatt SL, Crowley M, et al. A multicenter cohort study of treatments and hospital outcomes in neonatal abstinence syndrome. Pediatrics 2014;134:e527-34.

12 Finnegan LP, Connaughton JF, Kron RE, et al. Neonatal abstinence syndrome: assessment and management. Addict Dis 1975;2:141-58.

13 Zimmermann-Baer U, Nötzli U, Rentsch K, et al. Finnegan neonatal abstinence scoring system: normal values for first 3 days and weeks 5-6 in non-addicted infants. Addiction 2010;105:524-8.

14 Gold MS, Redmond DE, Kleber HD. Clonidine blocks acute opiatewithdrawal symptoms. Lancet 1978;2:599-602.

15 Yaster M, Kost-Byerly S, Berde C, et al. The management of opioid and benzodiazepine dependence in infants, children, and adolescents. Pediatrics 1996;98:135-40.

16 Hoder EL, Leckman JF, Poulsen J, et al. Clonidine treatment of neonatal narcotic abstinence syndrome. Psychiatry Res 1984;13:243-51.

17 Agthe AG, Kim GR, Mathias KB, et al. Clonidine as an adjunct therapy to opioids for neonatal abstinence syndrome: a randomized, controlled trial. Pediatrics 2009;123:e849-56.

18 Surran B, Visintainer P, Chamberlain S, et al. Efficacy of clonidine versus phenobarbital in reducing neonatal morphine sulfate therapy days for neonatal abstinence syndrome. A prospective randomized clinical trial. J Perinatol 2013;33:954-9.

19 Bada HS, Sithisarn T, Gibson J, et al. Morphine versus clonidine for neonatal abstinence syndrome. Pediatrics 2015;135:e383-91.

20 Benneyan JC, Lloyd RC, Plsek PE. Statistical process control as a tool for research and healthcare improvement. Qual Saf Health Care 2003;12:458-64.

21 Wu D, Carre C. The impact of breastfeeding on health outcomes for infants diagnosed with neonatal abstinence syndrome: a review. Cureus 2018;10:e3061.

22 McQueen K, Taylor C, Murphy-Oikonen J. Systematic review of newborn feeding method and outcomes related to neonatal abstinence syndrome. J Obstet Gynecol Neonatal Nurs 2019;48:398-407.

23 Charles MK, Cooper WO, Jansson LM, et al. Male sex associated with increased risk of neonatal abstinence syndrome. Hosp Pediatr 2017;7:328-34

24 Bhardwaj SK, Forcelli PA, Palchik G, et al. Neonatal exposure to phenobarbital potentiates schizophrenia-like behavioral outcomes in the rat. Neuropharmacology 2012;62:2337-45.

25 Bada HS, Bann CM, Whitaker TM, et al. Protective factors can mitigate behavior problems after prenatal cocaine and other drug exposures. Pediatrics 2012;130:e1479-88.

26 Larson JJ, Graham DL, Singer LT, et al. Cognitive and behavioral impact on children exposed to opioids during pregnancy. Pediatrics 2019;144:e20190514

27 Health Management Associates Institute on Addiction, California Perinatal Quality Care Collaborative, California Maternal Quality Care Collaborative. Mother and baby substance exposure toolkit. Available: NASToolkit.org [Accessed 28 Mar 2020].

28 Kunins HV, Bellin E, Chazotte C, et al. The effect of race on provider decisions to test for illicit drug use in the peripartum setting. $J$ Womens Health 2007;16:245-55.

29 Chasnoff IJ, Landress HJ, Barrett ME. The prevalence of illicit-drug or alcohol use during pregnancy and discrepancies in mandatory reporting in Pinellas County, Florida. N Engl J Med 1990;322:1202-6.

30 Terplan M, Minkoff $\mathrm{H}$. Neonatal abstinence syndrome and ethical approaches to the identification of pregnant women who use drugs. Obstet Gynecol 2017;129:164-7.

31 Grossman MR, Berkwitt AK, Osborn RR. Racial association and pharmacotherapy in neonatal opioid withdrawal syndrome: thinking beyond genetics. J Perinatol 2020;40:689-90.

32 Doerzbacher M, Chang Y-P. Supporting breastfeeding for women on opioid maintenance therapy: a systematic review. J Perinatol 2019;39:1159-64. 